\title{
INEQUALITIES INVOLVING CYLINDRICAL FUNCTIONS
}

T. B. CURTZ AND K. M. SIEGEL

Watson [1] derives the following equations for Bessel functions, valid for real positive $\nu$ and $0<x \leqq 1$.

$$
\begin{aligned}
& J_{\nu}(\nu x)=\frac{1}{\pi} \int_{0}^{\pi} \exp \{-\nu F(\theta, x)\} d \theta, \\
& J_{\nu}^{\prime}(\nu x)=\frac{1}{\pi} \int_{0}^{\pi} \frac{\theta-x^{2} \sin \theta \cos \theta}{x\left(\theta^{2}-x^{2} \sin ^{2} \theta\right)^{1 / 2}} \exp \{-\nu F(\theta, x)\} d \theta,
\end{aligned}
$$

where

$$
\begin{array}{r}
F(\theta, x)=\log \frac{\theta+\left(\theta^{2}-x^{2} \sin ^{2} \theta\right)^{1 / 2}}{x \sin \theta}-\cot \theta\left[\left(\theta^{2}-x^{2} \sin ^{2} \theta\right)^{1 / 2}\right], \\
0 \leqq \theta \leqq \pi .
\end{array}
$$

Using these relations Watson derives

$$
\begin{aligned}
J_{\nu}(\nu x) & \leqq \frac{\exp \{-\nu F(0, x)\}}{\left(1-x^{2}\right)^{1 / 4}(2 \pi \nu)^{1 / 2}}, \\
J_{\nu}^{\prime}(\nu x) & \leqq\left(1+x^{2}\right)^{1 / 4} \frac{\exp \{-\nu F(0, x)\}}{x(2 \pi \nu)^{1 / 2}}, \\
J_{\nu}(\nu) & <\frac{\Gamma(1 / 3)}{2^{2 / 3} 3^{1 / 6} \pi \nu^{1 / 3}} .
\end{aligned}
$$

One of the authors has shown that (3) may be replaced by an inequality which remains finite at $x=1$ [2]. Equations (3) and (4) have been extended to the case in which the argument is nearly equal to and larger than the order [3].

In this paper inequalities are derived relating $J_{\nu}(\nu x)$ and $J_{\nu}^{\prime}(\nu x)$ to the Bessel functions of equal order and argument, $J_{\nu}(\nu)$ and $J_{\nu}^{\prime}(\nu)$.

It is shown that

$$
\begin{aligned}
& J_{\nu}(\nu x) \leqq \exp \{-\nu F(0, x)\} J_{\nu}(\nu), \\
& J_{\nu}^{\prime}(\nu x) \leqq \frac{\left(1-x^{2}\right)^{1 / 2}}{x} J_{\nu}(\nu x)+x J_{\nu}^{\prime}(\nu) \exp \{-\nu F(0, x)\}, \\
& J_{\nu}^{\prime}(\nu)<\frac{3^{1 / 6} \Gamma(2 / 3)}{2^{1 / 3} \pi \nu^{2 / 3}} \approx \frac{0.41085}{\nu^{2 / 3}}
\end{aligned}
$$

Presented to the Society, April 16, 1955; received by the editors December 20, 1954. 
The right-hand members of (6) and (7) not only remain finite as $x \rightarrow 1$ but also approach $J_{\nu}(\nu)$ and $J_{\nu}^{\prime}(\nu)$ respectively. Equation (6) is an improvement of (3) when

$$
J_{\nu}(\nu)\left(1-x^{2}\right)^{1 / 4} \leqq 1 /(2 \pi \nu)^{1 / 2}
$$

while (7) is an improvement of (4) when

$$
J_{\nu}^{\prime}(\nu)<\frac{\left(1+x^{2}\right)^{1 / 4}-\left(1-x^{2}\right)^{1 / 4}}{x^{2}(2 \pi \nu)^{1 / 2}} .
$$

It can be observed

$$
\frac{\left(1+x^{2}\right)^{1 / 4}-\left(1-x^{2}\right)^{1 / 4}}{x^{2}(2 \pi \nu)^{1 / 2}}>\frac{0.199}{\nu^{1 / 2}} .
$$

Now comparing this with (8), (7) is an improvement of (4) for all $x$ whenever $\nu>78.5$ approximately.

Derivation.

PROOF OF (6).

$$
\begin{aligned}
& \frac{\partial}{\partial x} F(\theta, x)=-\frac{\theta-x^{2} \sin \theta \cos \theta}{x\left(\theta^{2}-x^{2} \sin ^{2} \theta\right)^{1 / 2}} \\
& \frac{\partial}{\partial x} F(0, x)=-\frac{\left(1-x^{2}\right)^{1 / 2}}{x}
\end{aligned}
$$

Now it is easy to show that

$$
\frac{\theta-x^{2} \sin \theta \cos \theta}{x\left(\theta^{2}-x^{2} \sin ^{2} \theta\right)^{1 / 2}} \geqq \frac{\left(1-x^{2}\right)^{1 / 2}}{x},
$$

that is

$$
\frac{\partial F(0, x)}{\partial x} \geqq \frac{\partial F(\theta, x)}{\partial x} .
$$

Integrating from $x$ to 1 and using the fact that $F(0,1)=0$

$$
F(\theta, x) \geqq F(\theta, 1)+F(0, x) \text {. }
$$

Therefore

$$
J_{\nu}(\nu x) \leqq \frac{1}{\pi} \int_{0}^{\pi} \exp \{-\nu[F(\theta, 1)+F(0, x)]\} d \theta
$$

whence follows (6).

Corollary. Using (5) we obtain 


$$
J_{\nu}(\nu x)<\frac{\Gamma(1 / 3)}{2^{2 / 3} 3^{1 / 6} \pi \nu^{1 / 3}} \exp \{-\nu F(0, x)\} .
$$

Proof of (7). Let

$$
L(\theta, x)=\frac{\theta-x^{2} \sin \theta \cos \theta}{\left(\theta^{2}-x^{2} \sin ^{2} \theta\right)^{1 / 2}} .
$$

Then

$$
L(\theta, x)=\frac{\theta\left(1-x^{2}\right)}{\left(\theta^{2}-x^{2} \sin ^{2} \theta\right)^{1 / 2}}+\frac{x^{2}(\theta-\sin \theta \cos \theta)}{\left(\theta^{2}-x^{2} \sin ^{2} \theta\right)^{1 / 2}} .
$$

Using

$$
\left(\theta^{2}-x^{2} \sin ^{2} \theta\right)^{1 / 2} \geqq \theta\left(1-x^{2}\right)^{1 / 2},
$$

and

$$
\begin{aligned}
\left(\theta^{2}-x^{2} \sin ^{2} \theta\right)^{1 / 2} & \geqq\left(\theta^{2}-\sin ^{2} \theta\right)^{1 / 2} \\
L(\theta, x) & \leqq\left(1-x^{2}\right)^{1 / 2}+x^{2} L(\theta, 1) .
\end{aligned}
$$

Hence

$$
\begin{aligned}
J_{\nu}^{\prime}(\nu x) \leqq & \frac{1}{\pi x} \int_{0}^{\pi}\left\{\left(1-x^{2}\right)^{1 / 2}+x^{2} L(\theta, 1)\right\} \exp \{-\nu F(\theta, x)\} d \theta \\
\leqq & \frac{\left(1-x^{2}\right)^{1 / 2}}{x} J_{\nu}(\nu x)+\frac{x}{\pi} \int_{0}^{\pi} L(\theta, 1) \exp \{-\nu F(\theta, x)\} d \theta \\
\leqq & \frac{\left(1-x^{2}\right)^{1 / 2}}{x} J_{\nu}(\nu x) \\
& \quad+\frac{x}{\pi} \int_{0}^{\pi} L(\theta, 1) \exp \{-\nu[F(0, x)+F(\theta, 1)]\} d \theta \\
\leqq & \frac{\left(1-x^{2}\right)^{1 / 2}}{x} J_{\nu}(\nu x)+x J_{\nu}^{\prime}(\nu) \exp \{-\nu F(0, x)\}
\end{aligned}
$$

and (7) is proved.

Corollary. Using (8) and (5)

$$
J_{,}^{\prime}(\nu x) \leqq \frac{\left(1-x^{2}\right)^{1 / 2}}{x} \frac{\Gamma(1 / 3)}{2^{2 / 3} 3^{1 / 6} \pi \nu^{1 / 3}} \exp \{-\nu F(0, x)\}
$$

$$
+\frac{3^{1 / 6} \Gamma(2 / 3)}{2^{1 / 3} \pi \nu^{2 / 3}} x \exp \{-\nu F(0, x)\} \text {. }
$$


Proof of (8). From the inequality

$$
\left(2 \theta^{2}-\sin ^{2} \theta\right) \cos \theta \leqq \theta \sin \theta \quad(0 \leqq \theta \leqq \pi) \quad[1, \text { p. 256] }
$$

it follows that $L(\theta, 1) / \theta$ is monotone decreasing $(0 \leqq \theta \leqq \pi)$. Hence

$$
L(\theta, 1) / \theta \leqq \lim _{\theta \rightarrow 0} L(\theta, 1) / \theta=2 / 3^{1 / 2} .
$$

Now

$$
J_{\nu}^{\prime}(\nu)=\frac{1}{\pi} \int_{0}^{\pi} L(\theta, 1) \exp \{-\nu F(\theta, 1)\} d \theta
$$

and since

$$
\begin{aligned}
& F(\theta, 1) \geqq 4 \theta^{3} / 9 \cdot 3^{1 / 2} {[1, \text { p. 259] },} \\
& J_{\nu}^{\prime}(\nu)<\frac{1}{\pi} \int_{0}^{\infty} \frac{2}{3^{1 / 2}} \exp \left\{-\nu \frac{4 \theta^{3}}{9 \cdot 3^{1 / 2}}\right\} d \theta<\frac{3^{1 / 6} \Gamma(2 / 3)}{2^{1 / 3} \pi \nu^{2 / 3}} .
\end{aligned}
$$

The right-hand side of the last inequality is the first term of Cauchy's asymptotic series for $J_{\nu}^{\prime}(\nu)$.

Further remarks. The recursion formulas can now be used as in [3] to produce inequalities for the case in which the argument of the Bessel function is greater than and nearly equal to the order.

This results in

$$
J_{\nu-1}(\nu x) \leqq\left[\frac{1+\left(1-x^{2}\right)^{1 / 2}}{x} J_{\nu}(\nu)+x J_{\nu}^{\prime}(\nu)\right] \exp \{-\nu F(0, x)\}
$$

valid for $1-1 / \nu<x \leqq 1$.

Once again this expression becomes an equality when $x=1$.

Similar inequalities may be derived for the Neumann function. For example, if $\gamma_{\nu}$ is defined by

$$
J_{\nu}(\nu)=-N_{\nu}(\nu) \tan \gamma_{\nu}
$$

it is shown in [1, p. 515] that $\gamma_{\nu}$ is an increasing function of $\nu$ for all positive $\nu$ and that $\lim _{\nu \rightarrow \infty} \gamma_{\nu}=\pi / 6$. Furthermore for so small a value as $\nu=12, \gamma=29^{\circ} 58-1 / 2^{\prime}$. Hence for $\nu \geqq 12$

$$
J_{\nu}(\nu) \geqq-.5766 N_{\nu}(\nu)
$$

or

$$
\left|N_{\nu}(\nu)\right| \leqq 1.73 J_{\nu}(\nu) .
$$

It is also shown in $\left[1\right.$, p. 487] that in the interval $\left(0, j_{v}^{\prime}\right)$, where $j_{v}^{\prime}$ de- 
notes the first zero of the derivative of $J_{\nu}(x), N_{\nu}(x)$ is negative and increasing. Hence for $j_{\nu}^{\prime} / \nu \geqq x \geqq 1$, a simple bound for $N_{\nu}(\nu x)$ is

$$
\left|N_{\nu}(\nu x)\right| \leqq\left|N_{\nu}(\nu)\right| \leqq 1.73\left|J_{\nu}(\nu)\right| \text {. }
$$

\section{REFERENCES}

1. G. N. Watson, A treatise on the theory of Bessel functions, 2d ed., Cambridge University Press, 1944.

2. K. M. Siegel, An inequality involving Bessel functions of argument nearly equal to their order, Proc. Amer. Math. Soc., vol. 4 (1953) pp. 858-859.

3. K. M. Siegel and F. B. Sleator, Inequalities involving cylindrical functions of nearly equal argument and order, Proc. Amer. Math. Soc. vol. 5 (1954) pp. 337-344.

UNIVERSITY OF MichigAN

\section{NEWTON'S METHOD IN BANACH SPACES ${ }^{1}$}

ROBERT G. BARTLE

In this note we show that if $f$ is a mapping between Banach spaces which is in class $C^{\prime}$ in the sense of Hildebrandt and Graves [4], ${ }^{2}$ then the equation $f(x)=0$ may be solved by an iterative process:

$$
x_{n+1}=x_{n}-\left[f^{\prime}\left(z_{n}\right)\right]^{-1} f\left(x_{n}\right), \quad n=0,1,2, \cdots,
$$

provided that the initial guess $x_{0}$ and the arbitrarily selected points $z_{n}$ are sufficiently close to the solution desired. Here the derivative is taken in the sense of Fréchet. If we let $z_{n}=x_{n}, n=0,1,2, \cdots$, we obtain the usual Newton process; if $z_{n}=x_{0}, n=0,1,2, \cdots$, we obtain what is sometimes called the modified Newton process. Naturally, in any application, the computer would determine the $z_{n}$ so as to minimize effort.

This result is closely related to recent theorems of Kantorovič $[5 ; 6 ; 7]$ and Mysovskih $[8 ; 9]$, although these authors assumed the existence and boundedness of the second Fréchet derivative of $f$. In turn for this assumption, they were able to establish more rapid convergence. Under the assumption of analyticity, Stein [10] eliminated explicit mention of the second derivative, which is desirable

Presented to the Society, December 29, 1954 ; received by the editors October 21, 1954 and, in revised form, December 9, 1954.

1 This paper was written while the author was on Contract nonr 609(04) with the Office of Naval Research.

${ }^{2}$ Numbers in brackets refer to the list of references at the end. 